\title{
Health Posts in Afghanistan
}

\section{Said Habib Arwal}

Community Based Health Care (CBHC), Afghanistan

"Corresponding author: Said Habib Arwal, Founder and National Coordinator for Community Based Health Care (CBHC), Afghanistan, Tel: 93700605071, Email: saidhabiba@gmail.com

Rec date: Oct 28, 2015, Acc date: Dec 08, 2015, Pub date: Dec 15, 2015

Copyright: (c) 2015 Arwal SH. This is an open-access article distributed under the terms of the Creative Commons Attribution License, which permits unrestricted use, distribution, and reproduction in any medium, provided the original author and source are credited.

Afghan Health System have a national Strategy that is Basic Package of the Health Service (BPHS), Health Post is the first step for providing of Primary Health Care Services.

Community-based basic health care services are provided by community health workers (CHWs) at their own homes recognized as community Health Post. Usually, a Health Post is staffed by a female and a male community health worker. If two (male and female) CHWs could not be available, one CHW may provide Health care services, but every effort should be made to provide two CHWs (a male and female) for every Health Post.

A Health Post will cover a catchment area of 1,000 to 1,500 people, which is equivalent to 100 or 150 families. In case of need, the coverage level of the Health Post can be changed. Health education and promotion of health habits in the community is the first and most important responsibility of the Health Post. In addition, the Health Post provides preventive care such as drug distribution and birth spacing devices, mothers' health care and limited curative services like diagnosis and treatment of common disease in children and adults.

The main responsibilities of a Health Posts are as follows:

Health education and changing of health habits of the community;

Referral of patients to health facilities;

Provision of first aid;

Treatment of common illnesses;

Community mobilization

The Community Health Worker is an important member of the health system, provides basic health care services from his/her home, which is recognized as a health post. The CHW is from the same area he/she is supposed to serve (familiar with the culture and language of the people), train for four- six months to deliver Basic Health Services. The CHWs are selected by the community they serve and supervised and monitored by the Community Health Supervisor from the nearest health facility.

Now there are about 15000 Health Posts, serving for rural population in Afghanistan, but there are still many un-served areas. Health posts through trained CHWs providing health care services to $45 \%$ sick children and covering $69 \%$ family planning, but some concerns are there about quality of the care they provide. CHWs workload is also a matter of concern, but not a major problem yet. CHWs motivation needs to be taken very seriously because they are volunteers. Recognition of their role in the health system, respect, honor, update of knowledge, and supportive supervision are important types of compensation. It is also very important to be specific about the different types of "incentives", including reimbursement of expenses, rewards for good performance or performance based financing to keep them in their volunteer role.

Urban CBHC program for poor urban populations, which is focusing on female CHWs, started in Kabul city, but other big cities are not covered yet. Nomad CBHC program for Nomad populations started but needs to have more CHWs for many un-served areas of Nomad populations

For establishment of a health post, in view of the following points a CHW should be identified and introduced by the community:

$B e$ a resident of the locality;

Be 20 to 50 years of age;

Be volunteer, active and interested to serve as $\mathrm{CHW}$;

Be respectable in his/her locality and enjoy the support of his/her community members;

Women should be encouraged to volunteer for being provided with training as $\mathrm{CHWs}$ (According to the guideline of the Ministry of Public Health, at least $50 \%$ of $\mathrm{CHWs}$ to be trained should be female.);

Basic literacy is preferable.

\section{Job Description of CHWs}

The community health worker (CHW) is a person (male or female) selected by the community according to selection criteria mentioned above. The CHW promotes healthy lifestyles in the community, encourages appropriate use of health services, and treats and refers common illnesses.

The CHW is accountable to the community Health Shura for performance and community satisfaction and is technically accountable to the community health supervisor (CHS). The CHW has the following responsibilities:

\section{A. Community Collaboration and Health Promotion}

\section{General responsibilities}

Actively participates in community meetings and major community events.

Actively works with mothers' groups to promote healthier homes and maternal and child health.

Encourages and mobilizes family/community participation in the immunization of children and women of child-bearing age.

Supports national initiatives at the village level and actively participates in all campaigns/activities e.g., National Immunization Days and surveillance for acute flaccid paralysis. 
Page 2 of 2

Promotes good nutrition practices and encourages early breastfeeding and exclusive breastfeeding of children less than six months of age.

Promotes use of Oral Rehydration Salt (ORS) and other homemade rehydration fluids for home management of diarrhea and dehydration.

Promotes hygiene and sanitation, and the preparation and use of safe drinking water.

Encourages couples to practice birth-spacing and receive family planning services.

9. Promotes psychosocial well-being and mental health in the community and raises awareness about prevention and identification of disabilities.

10. Creates awareness within the community and provides information on the dangers of addictive substances such as tobacco, naswar, opium, hashish, and alcohol.

\section{B. Direct Services}

Identifies and manages acute respiratory infections, diarrhea, malaria, and other common communicable diseases according to national protocols. Treats mild to moderate cases and refers complicated cases to the nearest health center.

Counsels patients on correct use of medications included in the CHW kit.

The CHW should create awareness among the community on how to prevent $\mathrm{TB}$ and should refer or accompany suspected cases to a health facility. Following completion by a tuberculosis patient of the first phase of treatment at the health facility, the CHW should ensure compliance of TB patients with the second phase treatment course in the community, based on DOTS.

Communicates the importance of antenatal and postnatal care. Distributes micronutrients and antimalarials to pregnant women according to national policy. Encourages the community to make regular and timely use of Maternal Child Health $(\mathrm{MCH})$ services.

Encourages the use of skilled birth attendants, where possible, and helps families make birth plans. Provides and teaches the use of a minidelivery kit (see Annex $\mathrm{C}$ for kit contents). Teaches family members to recognize the danger signs of complications of pregnancy and childbirth, and assists them in making preparations for emergency referral.

Distributes oral contraceptives and condoms to willing members of the target population according to national policy. Promotes LAM together with exclusive breastfeeding for the child's health during the first six months of a child's life. Administers first and follow-up injections of Depo Provera. Encourages interested families to seek long-term family planning methods at a health facility.

Provides first-aid services for common accidents at the family and community level.

\section{Management}

Meets regularly with the Shura to develop, implement, and monitor community action plans for health improvement.

Meets regularly with the community health supervisor to review reports and action plans, receive supplies, and for in-service training.

Regularly completes and submits the monthly Tally Sheets to the CHS for the HMIS.

Collaborates with and supports community midwife activities in his/her catchments area, including health promotion and pregnancyrelated referrals.

Develop a community map of the catchment area, knows the members of the community who are eligible to receive the health services.

Reports all deaths and informs the health facility of any disease outbreaks.

Manages the health post, maintaining supplies and drugs given to CHWs and reporting utilization of drugs and supplies.

Helps the CHS to form Shura-e-Sehee at the health post level.

\section{Conclusion}

Community based health care is a successful program in Afghanistan, which provide primary health care services as close as to the people homes through health posts by two male and female community health worker.

For now in Afghanistan, it is the best way to reach to the people homes, so it needs to be more strengthened and expanded. Also we should more focus on quality of health care services which provided by health posts

\section{Reference}

1. (2015) Ministry of public Health of Afghanistan, Community Based Health Care National Strategy 2020.

2. (2012) Ministry of public Health of Afghanistan, Community Health Workers training manual.

3. Ministry of public Health of Afghanistan, Community Based Health Care Department Documents 